\section{Zombie Journals: Designing a Technological Infrastructure for a Precarious Journal}

\author{
Daniel Paul O’Donnell, Carey Viejou, Sylvia Chow, Rumi Graham, \\ Jarret McKinnon, Dorothea Morrison, Reed Parsons, Courtney Rieger, \\ Vanja Spirić, \& Elaine Toth \\ University of Lethbridge
}

Kimberly M. Dohms

Canadian Wildlife Service

Paul Esau

Wilfrid Laurier University

Steve Firth

University of Helsinki

Kayla Ueland

University of Calgary

\section{Abstract}

Background The Meeting of the Minds graduate student journal is edited primarily by students from our Masters programme. This means that our editorial board is subject to high annual turnover and that our technological infrastructure and workflow needed to be easy to train for, accommodate differing levels of technological skill and editorial interest, and provide archiving that did not require a continuing interest in the journal by future generations of students.

Analysis This article provides a detailed and comparative account of the "off-the-shelf" systems and software used in developing the journal with an explanation of the rationale behind our choices.

Conclusion and implications The choices we made can be adopted by other journals interested in a low-cost, "future-proof" approach to developing a publishing infrastructure.

Keywords Publishing; Scholarly Communication; Academic Journals; Graduate Student Education

\section{CISP Press}

Scholarly and Research Communication

Volume 9, Issue 2, Article ID 0201296, 20 pages

Journal URL: www.src-online.ca http://doi.org/10.22230/src.2018v9n2e296

Received February 1, 2018, Accepted April 2, 2018, Published June 1, 2018

O’Donnell, Viejou, Chow, Dohms, Esau, Firth, Graham, McKinnon, Morrison, Parsons, Rieger, Spiric, Toth, Ueland. (2018). Zombie Journals: Designing a Technological Infrastructure for a Precarious Journal. Scholarly and Research Communication, 9(2): 0201296, 20 pp.

(C) 2018 Daniel Paul O’Donnell, Carey Viejou, Sylvia Chow, Kimberly Dohms, Paul Esau, Steve Firth, Rumi Graham, Jarret McKinnon, Dorthea Morrison, Reed Parsons, Courtney Rieger, Vanja Spiric, Elaine Toth, \& Kayla Ueland. This Open Access article is distributed under the terms of the Creative Commons Attribution Non-Commercial License (http://creativecommons.org/licenses/by-nc$\mathrm{nd} / 2.5 / \mathrm{ca}$ ), which permits unrestricted non-commercial use, distribution, and reproduction in any medium, provided the original work is properly cited.
VOLUME 9 / ISSUE 2/ 2018

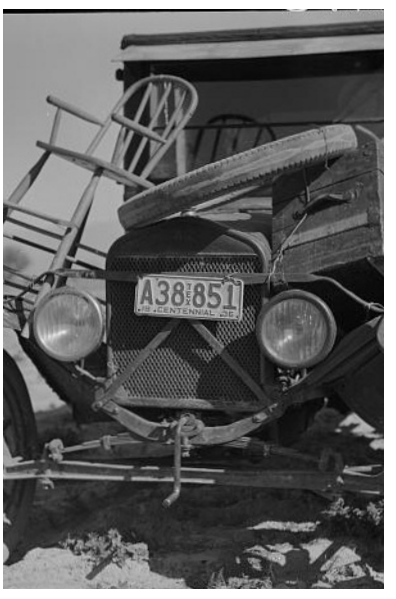

Photographer: Dorothea Lange (1937)

Daniel Paul O'Donnell is a Professor in the Department of English and University Library, Centre for the Study of Scholarly Communication at the University of Lethbridge. Email: daniel .odonnell@gmail.com

Carey Viejou is a Master of Arts student in the History department at the University of Lethbridge. She studies women's political activity in Alberta in the early 2oth century. Email: c.viejou@ uleth.ca.

Sylvia Chow is a graduate student at the University of Lethbridge. Email: sylvia.chow @uleth.ca 


\section{Scholarly and Research \\ Communication}

VOLUME 9 / ISSUE 2 / 2018

Kimberly M. Dohms is an Environmental Assessment Coordinator at the Canadian Wildlife Service. She completed her $\mathrm{PhD}$ in Evolution and Behaviour at the University of Lethbridge and is active in avian research, science outreach, and social activism. Email: kim.dohms @uleth.ca.

Paul Esau is a $\mathrm{PhD}$ student in History at Wilfrid Laurier University in Waterloo, Ontario.Email: paul.esau@ uleth.ca.

Steve Firth holds an MA in Philosophy and is a $\mathrm{PhD}$ candidate at the University of Helsinki in Finland. He is working on ethics especially those relating to disability and authors a philosophical blog entitled

"Hail Fellow Well Met," which seeks to advance philosophy and knowledge through accessible discussion of everyday dilemmas and events. Email: steven.firth@ uleth.ca.

Rumi Graham is a University Copyright Advisor and Graduate Studies Librarian. Email: grahry@uleth.ca.

\section{Introduction}

Meeting of the Minds (Graduate Student Association [University of Lethbridge], 2017a, 2017 b) is a graduate student-run scholarly journal at the University of Lethbridge in Alberta, Canada.

The journal was first proposed in the spring of 2015 as a venue for publishing abstracts and papers from a conference of the same name, which was established sixteen years previously by the university's Graduate Student Association (GSA). The goal of the journal was to provide students with experiential training in scholarly communication: in addition to authoring the papers that were published, students would act as the journal's managers and editors, and assist with and manage the peer review process. The journal would be run primarily on volunteer student labour, with several faculty members from the University of Lethbridge's Centre for the Study of Scholarly Communication (CSSC) serving as advisors. This labour, together with financial and in-kind support from the GSA and the Lethbridge Journal Incubator (an open access research and training project hosted at the CSSC), would help keep the costs down and ensure that the journal could publish student work without assessing Article Processing Charges (APC).

The intellectual and social aspects of the journal's development are discussed in a separate article (Esau, Viejou, Chow, Dohms, Firth, McKinnon, Morrison, Parsons, Rieger, Spiric, Toth, Ueland, \& O'Donnell, 2018). This article outlines the technological infrastructure and workflows that were developed to support the journal and, in particular, to address some of the key dangers that were foreseen in creating an open access, volunteer-led journal within a small, primarily masters-level graduate program. The result is a journal that, on the one hand, is both easy to train for and requires no annual maintenance, and, on the other, is easily discoverable and permanently and professionally archived. Because the editorial board is subject to high turnover as students complete their (usually) eighteen-to-twenty-four month programs, it was necessary to have a design that could be shut down and/or restarted easily, as editorial interest in the journal rises and falls over the years, without affecting the long-term preservation and discoverability of previously published articles.

\section{INSTITUTIONAL CONTEXT}

The University of Lethbridge ( $\mathrm{U}$ of $\mathrm{L}$ ) is a medium-sized (approximately 8,500 students), primarily undergraduate university with a strong research mandate (for statistical information about the university, see University of Lethbridge, 2017). Despite its size and undergraduate focus, the $\mathrm{U}$ of $\mathrm{L}$ is one of four Comprehensive Academic and Research Institutions (CARIs) established by the Province of Alberta to "conduct pure and applied research in a wide range of disciplines" and serve "the needs of learners interested in a comprehensive, research intensive environment" (Government of Alberta 2007). The other CARIs are the University of Alberta in Edmonton, the University of Calgary, and Athabasca University.

In keeping with its CARI status, the $\mathrm{U}$ of $\mathrm{L}$ has a small but growing graduate school (approximately 450 students) focused on masters-level study, particularly in the social sciences, humanities, and professional disciplines. Because the school is both

O’Donnell, Viejou, Chow, Dohms, Esau, Firth, Graham, McKinnon, Morrison, Parsons, Rieger, Spirić, Toth, Ueland. (2018). Zombie Journals: Designing a Technological Infrastructure for a Precarious Journal. Scholarly and Research Communication, 9(2): 0201296, 20 pp. 
comprehensive and small, instruction is typically carried out on a one-on-one, apprentice-style basis: students are encouraged during the admissions process to identify a supervisor, who is then responsible for signing off on most aspects of their subsequent program - from their choice of courses to the development of their theses. The small size and personal approach of the school also means that student numbers can vary greatly in individual departments from year to year as students enter and leave the program. It is not unusual in some departments for a "cohort" to consist of no more than one or two students (if that) in any one year, particularly in the social sciences and humanities, which enrol fewer graduate students than the sciences or professional faculties; although it is increasingly infrequent, in some years it is still the case that no students will be accepted for study in a given department.

\section{DESIGN PARAMETERS FOR TECHNOLOGICAL INFRASTRUCTURE}

\section{AND WORKFLOW}

This institutional context provided the main parameters to consider in the design of the technological infrastructure and workflow for the new journal.

1. High turnover. Because the $U$ of $L$ is a primarily masters-level school, a high turnover on the editorial board was anticipated. It was anticipated that many students would join the editorial board after they had established themselves in their program of study (i.e., in the second semester of their first year or, even more likely, the first semester of their second-and-final year). In many cases, this would mean that no more than a single year's participation from individual editorial board members could be expected, limiting the institutional memory.

2. Little opportunity for training. In addition to limiting the institutional memory, a high turnover means that training needs to be kept simple. Although the faculty advisors and support from the Journal Incubator and CSSC would provide some year-to-year continuity, neither had the capacity to support detailed training in journal-specific systems on an annual basis. This means that any systems would need to be closely related to software and processes students were already familiar with through their studies or that could be easily transferred to those studies.

\section{No guarantee that future generations of students would be interested in the} journal. When the Meeting of the Minds journal was first proposed in 2015, its namesake conference was being held for the sixteenth time. The fact that, as far as we are aware, nobody had previously proposed establishing a journal to accompany this conference suggested that we could not rely on future generations of students being as interested in the journal as we were. Rather, it seemed likely that we could expect interest to rise and fall over the years: for a year or two students might be highly interested in the journal, then for a few years there might be less interest, then perhaps interest might rise again or fall further. This meant that any systems and processes had to be able to be shut down gracefully, without removing or affecting the discoverability of the articles the journal had already published, should it prove impossible to find editors in a given year. And ideally, the system should be able to be started up again, should there be renewed interest in future years.

A fourth and fifth parameter involved the ethical obligation to student authors.

\section{Scholarly and Research}

\section{Communication}

VOLUME 9 / ISSUE 2 / 2018

Jarret McKinnon is a Master's student in the Department of Neuroscience at the University of Lethbridge. His research is geared toward the understanding of learning, memory, and cognitive processes and how these interact and contribute to semantic knowledge. Email: jarret.mckinnon@uleth.ca

Dorothea Morrison is an MA English student at the University of Lethbridge studying the intergenerational transmission of gender in the fiction of Margaret Atwood. Email: dorthea.morrison@ uleth.ca

Reed Parsons is a graduate student at the University of Lethbridge. Email: nr.parsons @uleth.ca

Courtney Rieger is a graduate student at the University of Lethbridge. Email: c.rieger@ uleth.ca

Vanja Spirić graduated from the Individualized Multidisciplinary MA program with a concentration in Sociology. She participated in the Lethbridge Journal Incubator program in 2016 and served as a board member on the Meeting of the Minds Graduate Journal in 2016-2017. Email: vanja .spiric@uleth.ca 


\section{Scholarly and Research}

\section{Communication}

VOLUME 9 / ISSUE 2 / 2018

Elaine Toth earned a BA with Distinction from the University of Lethbridge in April 2015. She is currently an MA Student in History. The working title of her thesis is Childhood Agency: Life Stories of Post-Second World War Dutch Immigrant Children to Alberta. Email: elaine.toth@uleth.ca.

Kayla Ueland is a second year law student at the University of Calgary. Email: kaueland@ ucalgary.ca.
4. Articles published by the journal needed to be permanently available, regardless of the current status of its editorial board. The students who submitted articles to the journal were doing so presumably because they wanted to acquire experience with the publication process and because they wanted to make their research known to a wider audience. Such students would presumably also want to include their publications in the journal on their curricula vitae (CVs), refer to them in their theses and subsequent publications, and use them, in many cases, when applying for further graduate study or research jobs. This presented an obligation to ensure that the authors' articles remained discoverable and citable no matter what changes occured in editorial interest or support from the faculty, CSSC, or GSA.

5. Need to ensure professional archiving and discoverability. Finally, it was made clear by students and faculty members that any established system had to use professional archiving and discoverability tools. The journal needed to have an International Standard Serial Number (ISSN) and, even more importantly, articles needed to have some kind of permanently resolvable identifier, such as a Digital Object Identifier (DOI).

\section{Available systems}

These parameters ruled out some common approaches to establishing a scholar-led journal.

\section{Open Journal Systems}

A common system used for supporting the publication of open access journals is Open Journal System (OJS). This is open-source software and hence, in principle, free to acquire, set up, and operate. On the other hand, it is specialized software whose setup, operation, and maintenance requires correspondingly specialized knowledge (e.g., arranging and/or setting up an internet server and the installation of the application as well as installing patches and updates). In order to use OJS, you need either somebody on staff who is comfortable administering server-based software, or to contract with (and in most cases pay) a service to do this maintenance for you. It is also not a "publish and forget" system; as a complex, continuously developing product, it can become susceptible to hacking, spam, and other dangers if it is not maintained regularly (the Journal Incubator, which operated its own installation of Open Journal Systems for several years, was regularly spammed when it failed to update its system on time). ${ }^{1}$

Taken together, these issues are incompatible with parameters one through four: there is no way of guaranteeing that the expertise required to set up and maintain the system would be available in any given year (or remain available given the expected turnover in the board), and the need to maintain the system after it has been established (or contract with a service to do this maintenance) means relying on the willingness of future generations of students (or the GSA, CSSC, or faculty members) either to devote effort to carrying out this maintenance or to pay for a service to do the work for them. Martin Eve (2012) estimated that maintaining an OJS installation, including fees for discovery aids such as DOIs, cost around \$225 per year in 2012. If interest in operating and maintaining the journal collapsed entirely in some future year, the current students' publications would run the risk of being permanently lost.

O’Donnell, Viejou, Chow, Dohms, Esau, Firth, Graham, McKinnon, Morrison, Parsons, Rieger, Spirić, Toth, Ueland. (2018). Zombie Journals: Designing a Technological Infrastructure for a Precarious Journal. Scholarly and Research Communication, 9(2): 0201296, 20 pp. 
A second problem with OJS involves the question of training: as a specialized journal management system, OJS is not a tool students would otherwise use every day in their research and coursework; this means that a journal relying on OJS needs to train specifically for it, rather than relying on students being able to transfer their knowledge of other systems to and from their day-to-day work. This implies continuity (somebody needs to understand or research how the system works in order to train others) and takes time. At the Journal Incubator, for example, students are generally trained on OJS by students with previous experience and learn by following a few articles through the workflow before they are assigned one to manage on their own (on the incubator approach, see Cowan, 2013; O'Donnell, Hobma, Cowan, Ayers, Bay, Swanepoel, Merkley, Devine, Dering, \& Genee, 2015; Swanepoel, Kehoe, Hohner, Shepstone, Vanderjagt, Wakaruk, Waller, \& Winter, 2015). In this journal, we expected very little year-to-year continuity and certainly could not guarantee that what continuity there was would involve the most technically competent students in any one cohort. The need to train on a specialized system, therefore, was a significant impediment to adopting OJS.

This training issue extends to production tasks. While OJS can be used to manage journal workflows and publish and archive articles, it is not itself a production system. The production of publishable PDF or HTML output from author manuscripts (usually in Word) takes place outside the Open Journal System in most cases, meaning that students need to be trained in such production tasks as well as day-to-day workflow management within OJS.

Finally, there was the issue of professional archiving and discoverability. Open Journal System is a workflow management and publication system, not a hosted archive service or discoverability system. While it can be used to publish articles and maintain archives, long-term preservation depends on the willingness of the publisher to pay for server space. Likewise, while it greatly streamlines and professionalizes the process by which articles are submitted, reviewed, and published, the core publication and discovery infrastructure (such as URLs, ISSNs, DOIs, and relationships with indexing and aggregation vendors) must be arranged by the journal managers outside the application.

\section{Blog/CONTENT MANAGEMENT Systems}

Another approach to scholar-led journal publishing is to use commercial blogging or content management software - perhaps, most commonly, through a service such as Wordpress (see, for example, Leubsdorf, 2011). Such systems have the advantage of a huge user base, and intuitive, consumer-friendly interfaces (see Ghosh, 2014, in the context of academic publishing). They are already familiar to many students and also represent a skill that is transferable to or from other aspects of their research and teaching.

On the other hand, such systems are not specifically designed to handle journal workflows in their stock setup (see sbstarr, Laxmin, \& PixelatorOfTime, 2015). To the extent they rely on plugins or third-party add-ons, moreover, they are susceptible to problems with these third-party providers, including bugs, viruses, and the possibility

O’Donnell, Viejou, Chow, Dohms, Esau, Firth, Graham, McKinnon, Morrison, Parsons, Rieger, Spiric, Toth, Ueland. (2018). Zombie Journals: Designing a Technological Infrastructure for a Precarious Journal. Scholarly and Research Communication, 9(2): 0201296, 20 pp.
VOLUMe 9 / ISSUE $2 / 2018$ 


\section{Scholarly and Research}

\section{Communication}

VOLUME 9 / ISSUE 2 / 2018 that these providers will cease development and support (e.g., Solvitor LLC, n.d.). Wordpress and similar sites also still require a commitment to support a URL and other discovery aids (leaving annual costs), or, if a free hosting option is chosen through a provider such as Wordpress.com, exist at the sufferance of the hosting service. The "terms of service" at Wordpress.com, for example, allow the site owner to "terminate your access to all or any part of our Services at any time, with or without cause, with or without notice, effective immediately" (Automattic Inc., 2014), imperilling long-term preservation. And finally, as John W. Maxwell $(2007,2009)$ has shown implicitly in his studies of small Canadian magazines, such ad hoc systems can rapidly become quite complex as additional systems and platforms are brought in to support specific workflow management problems.

Such systems address parameters one through three, in that they are well-known, easy to train for (assuming they avoid the type of system creep Maxwell [2007, 2009] illustrates), involve transferable skills, and, using the free hosting option (provided one's account is not suddenly terminated), may end up being maintained without effort or costs indefinitely. But they do not satisfy the fourth or fifth requirement: their archiving is not guaranteed, and they are not automatically integrated with sectorstandard discovery systems.

\section{Commercial PlatForms}

A third option involves subscribing to a commercial or nonprofit service: either through a company such as Scholastica or by joining a consortium such as the Open Library of the Humanities (Open Library of Humanities, n.d.; Scholastica, n.d.). In the case of a commercial service, the subscription requirement may violate the "publish and forget" requirement: in most cases, ongoing preservation and discoverability requires somebody to be willing to pay for the service to continue. In the case of a service such as the Open Library of the Humanities, on the other hand, the journal would need to first prove itself (including its long-term viability) before it could apply for admittance. Neither approach is suitable for a new student journal that may or may not publish regularly and is almost certainly going to be of lower prestige (due to its different focus and goals) than professional disciplinary journals. If the journal continues to prosper, alignment with the Open Library might become possible in the future - although this brings with it obligations that might be incompatible with the kind of turnover and potential change in interest envisioned for the journal. In the short term, however, it is an unlikely option.

\section{“GREEN" OPEN ACCESS/INSTITUTIONAL REPOSITORIES}

A final option involves largely giving up on "Gold” (i.e., publisher organized) open access (for this terminology, see Suber, 2013) altogether and instead encouraging, arranging, or assuming that authors will independently archive their publications. Or alternatively, arranging with an institutional repository (IR) to serve as a backup for the journal - archiving publications as they appear and providing a discoverable location for them should the journal shut down, or if any of the possible disruptions that have been considered take place (loss of URL, failure to maintain the platform, etc.). As JeanGabriel Bankier and Irene Perciali (2008) point out using the specific example of student journals, IRs could themselves be used to publish rather than as a backup; this

O’Donnell, Viejou, Chow, Dohms, Esau, Firth, Graham, McKinnon, Morrison, Parsons, Rieger, Spirić, Toth, Ueland. (2018). Zombie Journals: Designing a Technological Infrastructure for a Precarious Journal. Scholarly and Research Communication, 9(2): 0201296, 20 pp. 
partially anticipates our solution of using Zenodo, but, more importantly, depends on policies and an approach to deposits that have not yet been implemented at the University of Lethbridge.

This approach "satisfies" design parameters one through four, largely by assuming they will not be met and instead planning for their failure. Assuming, encouraging, or arranging for the publications to be preserved by somebody else relieves the need to worry about the quality or durability of the systems used for publication: provided all of the articles are actually preserved by third parties, it would not matter if the platform was unstable, or if future generations of students lost interest in or failed to maintain the work; it would also make training considerably easier, since the only goal would be to provide output formats that other repositories could archive.

The downside is that it fails to satisfy clearly the fifth requirement - the need to ensure professional archiving and discovery. While the articles that were committed to institutional repositories presumably would be curated professionally, there would be no guarantee that all articles would be preserved in this fashion (not all graduate students are necessarily aware of what is involved in arranging for the archiving of their work). Unless specific arrangements were made with a repository, moreover, there would be no guarantee that all articles would be able to find a home, even if every author was equally diligent in seeking one: different institutions have different policies with regard to the archiving of student (and even faculty) work; some students might not be in institutions that would allow them to preserve their work in this way.

And finally, of course, there would be the lack of coherence. If authors are relied on to preserve their own work and pay no attention to the survival of the journal as a platform, there would be very little difference between the articles published in the journal and the many self-published pieces that make up the burgeoning "grey literature." While journal articles would have been peer reviewed/mentored, there would be very little evidence of the journal that (once) stood behind their publication. Different articles would be stored in different locations with potentially different metadata and evidence of quality control. Perhaps, especially for graduate students who are beginning their academic careers and for whom individual publications have an inordinate weight on their CVs, this approach seems to ignore the ethical duty to provide authors with a professional-looking, discoverable, and citable output. The lack of an actual "journal" to pass on - i.e., a site with content - seems likely to make maintaining editorial continuity and enthusiasm across cohorts even more difficult and unlikely.

\section{The approach: Use "off-the-shelf" systems and software}

As the above suggests, our review of existing systems and approaches led us to the conclusion that none were ideally suited to the requirements of something that might quickly become a "zombie." This was particularly true of the consistent failure of these approaches to meet the fourth and/or fifth design parameters (the ones we considered the most important, ethically speaking): any journal we set up had to be capable of ensuring the permanent availability of our authors' work, without requiring the intervention or support of future generations of graduate students; and any journal we

O’Donnell, Viejou, Chow, Dohms, Esau, Firth, Graham, McKinnon, Morrison, Parsons, Rieger, Spiric, Toth, Ueland. (2018). Zombie Journals: Designing a Technological Infrastructure for a Precarious Journal. Scholarly and Research Communication, 9(2): 0201296, 20 pp. 


\section{Scholarly and Research}

\section{Communication}

VOLUME 9 / ISSUE 2 / 2018 established had to be able to offer our authors free, long-term, professional archiving, and discoverability. While it would be nice to have a system that was easy to train for, this was less important than the need for something that would guarantee the easy availability of student work, even if the journal was subsequently abandoned.

As a result, we decided to build our own infrastructure, restricting ourselves entirely to "off the shelf" services and software:

- For our editing/production software, we used existing, widely used, consumeroriented word processors - Microsoft Word and Libreoffice.

- For our storage and discoverability systems, we built a community on Zenodo, a free, EU- and CERN-supported data and preprint repository that provides DOIs for all deposits and guarantees the long-term preservation of research data entrusted to it.

- For dissemination, we used two methods: the built-in "community" function at Zenodo and, out of a concern that this would insufficiently emphasize the connection between the journal and the $\mathrm{U}$ of $\mathrm{L}$, a Wordpress site at a custom URL that used our Zenodo repository as a document server (in retrospect, however, this second site turned out to be less valuable than we imagined: the Zenodo community is more than sufficient to brand the journal as ours).

This is, of course, quite similar to the second and fourth methods investigated above (using commercial blogging software and the "green" self-archiving method). There are, however, several important differences:

- Instead of relying on our authors to find their own archiving (i.e., "green" open access), the journal itself arranged for the long-term, open preservation of its output (i.e., a form of "gold" open access).

- Instead of treating the repository as a "backup" of the journal, we used Zenodo as the publication platform. In other words, Zenodo became the host for the journal, allowing us to keep the articles together and ensure some consistency across individual outputs.

- While we established a Wordpress site to brand the journal, this site was secondary to the Zenodo repository. For example, the DOIs assigned to the articles all resolve to the Zenodo site, rather than the Wordpress site. Also, the Zenodo site acts as the document server that supplies the PDFs to the Wordpress site (see Figure 1). This means that the journal will survive as a coherent collection even if our custom URL and Wordpress site are allowed to lapse.

In the end, this approach satisfied all five design parameters, resulting in an easy to use and easy to train for journal that is both "publish and forget" and provides professional discovery and archiving. Even if there is a complete collapse in student interest in maintaining the journal, the articles that are already published will remain available and associated with the journal. 
Figure 1: The Meeting of the Minds production and publication infrastructure

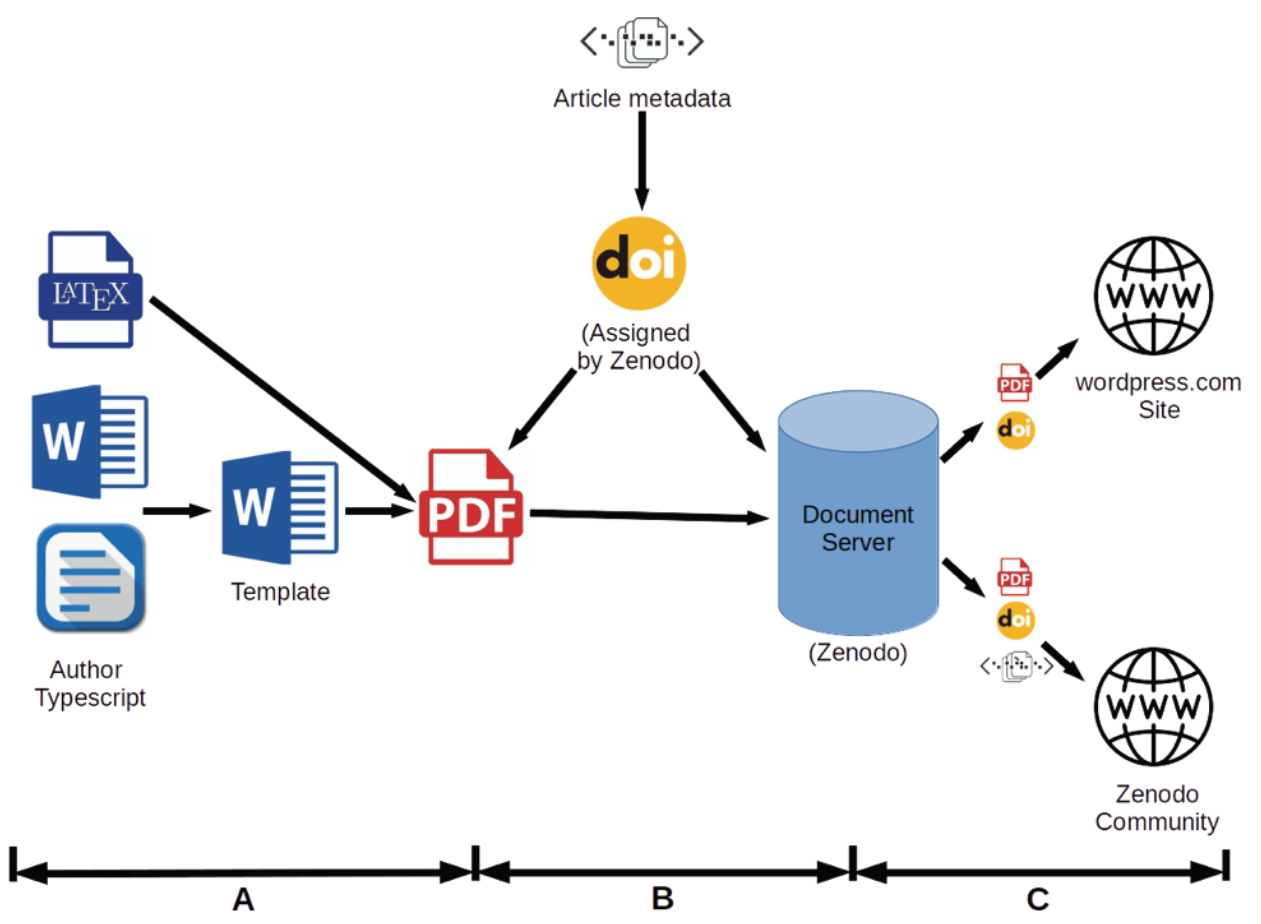

The Meeting of the Minds production and publication infrastructure consists of three main steps and processes.

A. Copy and galley production. This encompasses the steps required to get an accepted manuscript to proof (in this case, PDF).

B. Metadata registration. This involves the registration and publication of metadata about individual articles, ensuring their future discoverability and accessibility.

C. Publication and archiving. The infrastructure used to deliver articles to users and preserve them for future use.

The following sections explore the precise details of the infrastructure, acknowledging the strengths and weaknesses.

\section{A. Copy and galley production}

Meeting of the Minds publishes articles as PDFs (see examples at Graduate Student Association [University of Lethbridge], 2017b). Because the articles it publishes are usually among the authors' first publications, and hence likely to play an important role in early career decisions, the editorial board felt strongly that the result had to have a "professional" appearance - that is to say that the mise-en-page and apparatus of the article had to be generically conservative and match those stereotypically associated with scholarly publication in professional disciplinary journals.

\section{SUbMisSion FORMATS, STYLE GUIDES, AND CITATION}

An early question had to do with different disciplinary standards for manuscript submission: which document types would be accepted, what style guide(s) would be followed, and what citation styles would be used. In most of the humanities and the social sciences, for example, manuscripts are most commonly submitted as word 


\section{Scholarly and Research}

\section{Communication}

VOLUME 9 / ISSUE 2 / 2018 processor files (particularly Microsoft Word), whereas in most of the sciences and some humanities and social sciences disciplines, the standard format for submission is LaTeX. Stylistic expectations also vary widely among disciplines. In many disciplines, articles are expected to have a standard format and outline. In others, they are built organically according to the argument and can vary greatly in structure and form. Some disciplines place a heavy emphasis on literary style; others prefer highly technical or compact writing. And of course, individual disciplines (and even subdisciplines) have firm expectations with regard to citation style: MLA, MHRA, or Chicago AuthorDate in literary studies; Chicago or Turabian note style in history; APA in many social sciences; and Harvard and other styles in the natural sciences (in discussion of these issues, one faculty advisor told the story of an author who withdrew an article from a collection rather than convert his citations from footnotes to parentheses).

Since the goal of this journal was to assist in the professional development of students in the $\mathrm{U}$ of $\mathrm{L}$ graduate school by providing them with an opportunity to practice scholarly publishing, it was important to follow the norms of the students' home disciplines as much as possible. It was decided, therefore, that neither a "house style" for citation nor submission in a particular document format were required. Students in the social sciences and humanities could submit their article in common word processor formats such as Word and Libreoffice (several members of the editorial team were open source advocates and/or Linux users and argued strongly against restricting submission to Word only); students in the natural sciences could use LaTeX. Faculty advisors would be asked to comment if either the document format or citation styles conflicted with disciplinary norms. But otherwise, we would restrict the copy editing to ensuring consistency within a student's chosen citation system.

In terms of mise-en-page, this decision meant that we anticipated the journal would have two main types of articles: those formatted from word processor documents, which would follow the single-column style now common in humanities journals like the PMLA; and those formatted from LaTeX files, which would follow the double column style common in science journals like PLoS. It also meant that there might be little consistency in citation style across articles in any one issue if the submissions came from a range of disciplines.

While this lack of consistency across articles might traditionally be understood to reflect poorly on the publisher, we believed it would likely be largely unnoticed in the journal, given the wide disciplinary range it aimed to cover. The days in which readers find scholarly articles solely by going to a specific journal issue and selecting relevant literature are long past. Contemporary researchers often use search engines to hone in on specific articles without regard to the source journals. In our case this concern turned out to be moot, as our first issue consisted entirely of articles from the social sciences and humanities rather than the broader range of disciplines that was originally hoped for. Given this, we felt that readers would more likely be disconcerted by an article that failed to follow disciplinary norms in citation and style than by an explicitly multidisciplinary journal that contained articles following different disciplinary norms and styles.

O’Donnell, Viejou, Chow, Dohms, Esau, Firth, Graham, McKinnon, Morrison, Parsons, Rieger, Spirić, Toth, Ueland. (2018). Zombie Journals: Designing a Technological Infrastructure for a Precarious Journal. Scholarly and Research Communication, 9(2): 0201296, 20 pp. 


\section{PROOF PREPARATION}

Since LaTeX documents are commonly used to produce PDF output and authors submitting in LaTex are expected generally to prepare their own galleys, it was anticipated that we would not be involved in the production of proofs for articles in the sciences, other than checking the proofs that were prepared for us.

In the case of articles submitted from the humanities and social sciences, we expected to be more involved. There are various ways of generating PDF proofs from word processor documents, including online services, desktop publishing applications, word processor-LaTeX plugins, and conversion via an intermediate format, such as XML. In keeping with the second requirement (i.e., that any systems used would be closely related to software and processes students were already familiar with through their studies or that could be easily transferred to those studies), we decided to train student editors to enforce consistency of appearance and produce PDFs for distribution using word processor templates and styles instead of using specialized typesetting software or services.

1. We developed a Word template containing the basic mise-en-page and styles for use in all humanities and social sciences submissions (Viejou \& O'Donnell, 2018).

2. After copy editing was finished on a given article, the contents of that article were pasted into a new instance of the journal template.

3. The student typesetter then formatted the article's sections to give the document a structure using word processor styles: "body text" for paragraphs; "quotation" for block quotations; "heading 2" for first-level subheadings; and so on (for an archived copy of the manual, see O'Donnell \& Viejou, 2017).

4. The file was then saved as a PDF and sent to the author for proofing.

The advantage of this process is that it trains students in skills that are directly transferable to their other activities: word processor styles and templates can be used by students in their own work as, for example, they format their theses for final submission. While Word is not the best typesetting program, it is common software, widely available, and widely supported: this keeps the training relatively easy and applicable to other tasks in students' daily lives. Experience in the Journal Incubator suggests that this is among the most valued (and indeed extensible) aspects of the training students receive in that program.

\section{B. Metadata registration}

Authors stated that the journal had to use professional discovery and identification aids such as DOIs and ISSNs (we did not consider enforcing author use of ORCID, though in retrospect this might have been a beneficial policy assisting students in establishing themselves in academia). While some of this interest was indeed to ensure that their work was available for citation, it also gave the impression - particularly in communication with some supervisors - that the presence of such aids were also understood as an indication of our own seriousness as a publication venue (one supervisor told us that he would discourage any student from publishing in a journal that did not have an ISSN). 


\section{Scholarly and Research}

Communication

VOLUME 9 / ISSUE 2 / 2018
ISSNs are easy to obtain in Canada (Library and Archives Canada, 2017). We applied and received one for free using the Library and Archives Canada website.

More difficult, but in our view more important to our authors, were DOIs. We decided early on that the ability to publish articles with DOIs was very important to us. In addition to satisfying author generic expectations (i.e., the presence of DOIs conforms with author expectations for a "serious" journal), DOIs also help with dissemination, discovery, and preservation. All the major citation management, repository, and CV systems (including Endnote, Paperpile, Zotero, Academia.edu, Research Gate, the Canadian Common CV, and ORCID) read and import DOIs. They are also a mechanism for avoiding the problem of broken URLs as long as they have been properly transferred, DOIs always resolve to the correct URL for a given object.

The main issue with DOIs is how to create and assign them. Attaching a DOI to an article requires registering its metadata with a registration authority. It is possible to become a member of a service such as Crossref and acquire the authority to register DOIs directly (e.g., Hendricks, n.d.). But this costs money, requires some maintenance (especially if URLs are changed), and involves some not-easily-transferable training or expertise (violating parameters one through four). While this approach would work for even small scholar-led professional journals, it was inappropriate for this journal, where the interest (and institutional knowledge) necessary for maintaining the system could vanish from one year to the next.

An alternative commonly used by individuals is to publish with a site that issues DOIs for submitted documents (e.g., Zenodo, Figshare, Researchgate, Academia.edu). In such cases, the DOI refers to the document version stored in the selected site. Posting the publisher-version of an article to one of these sites can thus result in a document that has two or more DOIs: one from the original publisher and one from each site in which it is deposited.

This was, of course, one of the attractions of the "fourth" or "green" approach to preserving the articles that was considered and rejected. Although OPUS, the University of Lethbridge institutional repository, currently does not assign DOIs to submissions, most repositories do. While these services were originally intended to assist in the publication and discoverability of "grey literature" - datasets, preprints, reports, and unpublished manuscripts - they can also be used to register individual articles in a journal such as this one.

As discussed above, in many cases repositories and social sites such as Academia.edu and Researchgate are ill-equipped to serve as the primary publication platform for a journal. For instance, preserving an article in a commercial network such as ResearchGate or Academia.edu or a commercial repository such as Figshare can result in a lack of internal coherence for the journal as a whole. Such sites are focused on providing access to individual objects, rather than the preservation of collections of objects (a journal issue, by contrast, is by definition a collection of research objects aimed at long-term preservation). In the cases particular of the commercial social networks Academia.edu and Researchgate, moreover, the terms and conditions

O’Donnell, Viejou, Chow, Dohms, Esau, Firth, Graham, McKinnon, Morrison, Parsons, Rieger, Spirić, Toth, Ueland. (2018). Zombie Journals: Designing a Technological Infrastructure for a Precarious Journal. Scholarly and Research Communication, 9(2): 0201296, 20 pp. 
explicitly allow the owners to remove content at any time and without notice (Fortney \& Gonder, 2015; Figshare is an exception among commercial providers in that it has developed partnerships to ensure the long-term survival of submissions to its collection). Furthermore, their DOIs as a rule are branded for the repository rather than the journal: they invariably have the repository name in the ID and do not allow for branding by another entity (e.g., a Figshare DOI: 10.6084/m9.figshare.3413821.v1).

A partial exception to these issues (aside from that of branded DOIs) is Zenodo (n.d.), an open science, open data service of the European Union OpenAIRE project and CERN. On the one hand, Zenodo is similar to many commercial and institutional repositories in that it accepts deposits of scholarly and scientific material with very few restrictions; registers the metadata for these deposits; and assigns them DOIs. On the other hand, Zenodo differs from many commercial and institutional repositories in that it has adopted a community model for organizing individual objects and has instituted several version and access control mechanisms explicitly designed to encourage the use of the platform for publishing (see Zenodo, n.d., for a brief discussion of these features).

This combination provides a journal like ours with a means of publishing individual articles in an archivally responsible and discoverable way as well as a curating a collection of such objects through their inclusion in a Zenodo community. The latter identifies the relationship among them as a scholarly artifact almost as much as the objects themselves. ${ }^{2}$

Registering objects with Zenodo is a matter of filling in a selfexplanatory form and uploading the object to the repository (see Figure 2). The form allows the user to "pre-reserve" a DOI -i.e., hold a DOI number in anticipation of the registration - so that this same DOI can be added to the object itself. This feature allowed for the inclusion of the reserved DOI in the colophon in the footer of the front page (see any of the examples in Graduate Student Association [University of Lethbridge], 2017b). At the time we were uploading our articles, Zenodo did not support
Figure 2: The Zenodo deposit form showing communities
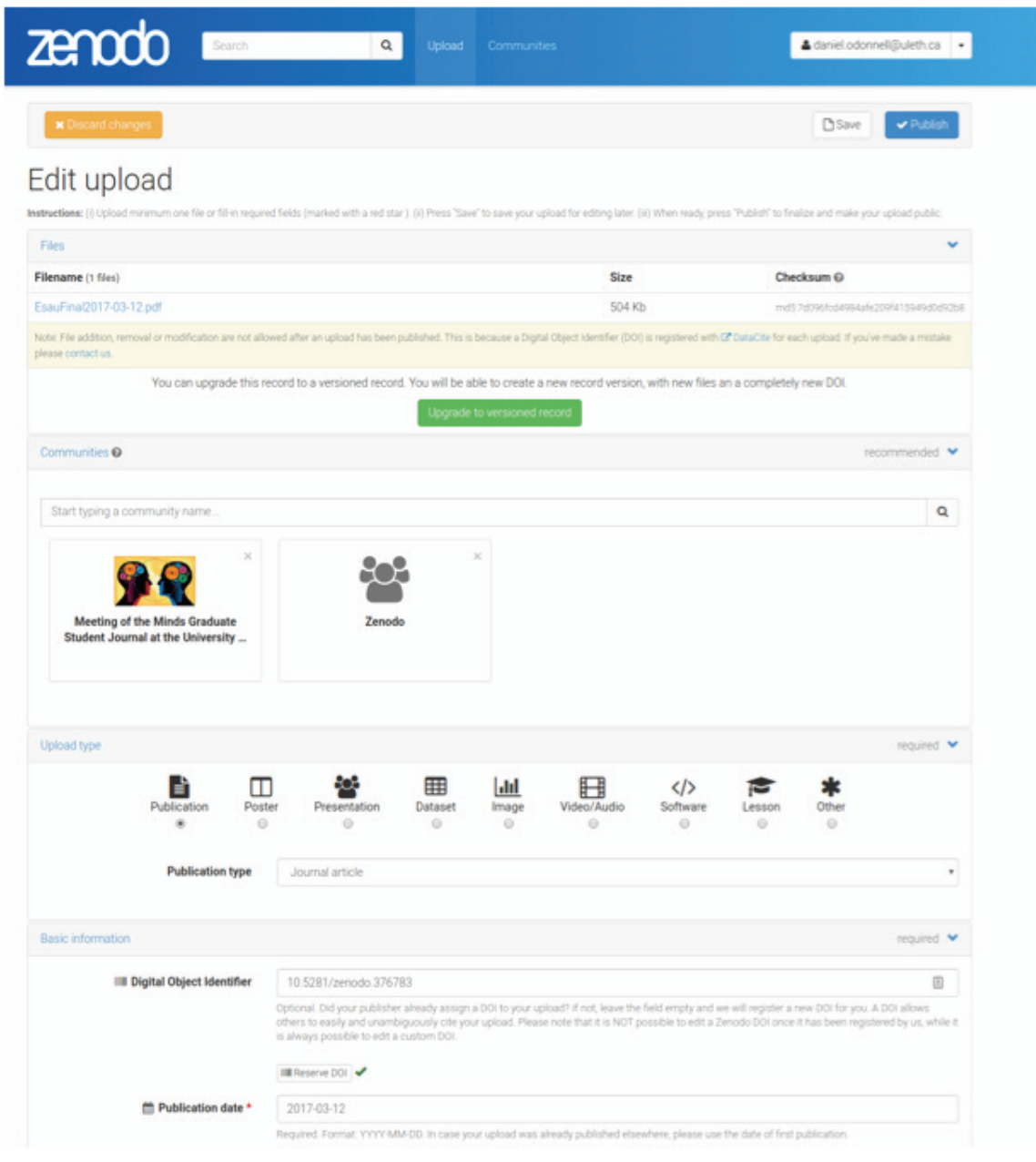


\section{Scholarly and Research}

\section{Communication}

VOLUME 9 / ISSUE 2 / 2018

versioning, meaning that any submission to the repository was considered final as soon as the "Publish" button was clicked. The system has since developed a method for associating different versions of the same object.

During or after the publication process, depositors can request inclusion in one or more Zenodo communities. In the case of the Meeting of the Minds journal, student editors uploaded the articles to Zenodo and requested that the object be associated with the journal community. In this case, the connection was made automatically. If somebody without administrative rights to the community requests a connection, the object is referred to the community administration for archiving.

Once an object is published, it is available from the Zenodo site. If it has been accepted to a Zenodo community, it can be accessed either directly or via that community. The landing page for an individual object provides some metadata (title, author, abstract, keywords, communities, DOI, etc.), and, if the format is supported, a thumbnail view of the file itself (Figure 3). The actual object - i.e., the PDF, database, image, etc. described by this metadata

Figure 3: Screenshot showing entry for a typical article in the Meeting of the Minds Journal ${ }^{3}$

\section{Zenodo \\ March 12,2017 \\ a \\ Selling Out to the Saudis: A Historical Analysis of Stephane Dion's Apology for Canadian Military Export Controls}

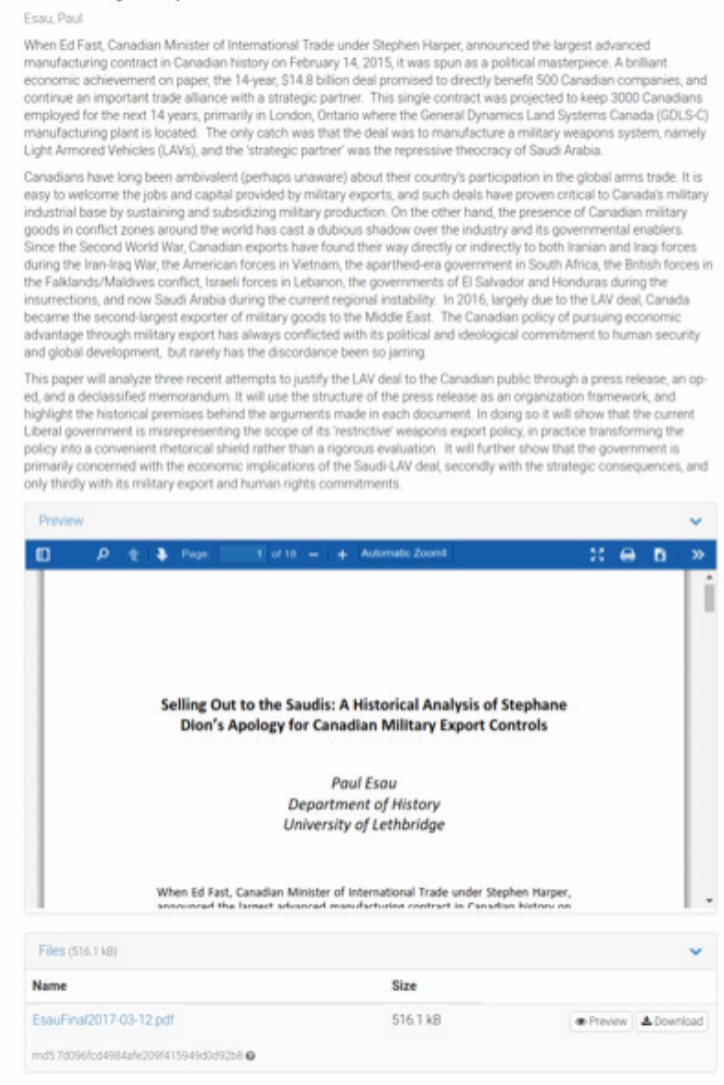

- is directly available via a permanent URL.

\section{Publication and archiving}

The third part of our production and publication infrastructure involves dissemination. In this step, two methods were used.

\section{ZENODO}

The first (and core) method is the Meeting of the Minds Zenodo Community (Graduate Student Association [University of Lethbridge], 2017b) (see Figure 4). This is an attractive, automatically generated website that looks similar to many journal interfaces (including some presentations of OJS) and provides access to all publications of the journal.

Articles published to this site are permanently archived at no cost to the journal or the author. Each article is presented with core metadata (date, genre, licence, author, abstract) and colophon information for the journal itself. If a citation to an article published in the journal contains a DOI, the 
DOI resolves to this site. There is a search function that allows users to search this specific community for content - allowing, for example, for a user to find a specific article even if all they know are some key terms and the journal name.
Scholarly and Research

Communication

VOLUME 9 / ISSUE 2 / 2018
The only negative aspect of this presentation is that the site provides no method for subdividing content within a community. Unlike other journal publication platforms (e.g., OJS), there is no mechanism for grouping articles into issues, themes, or topics. Instead, Zenodo treats content posted to a community essentially as blog posts: they are laid out in reverse chronological order, with the most recent postings at the top, regardless of relevance or topic. (In Figure 4, for example, workflow management documents intended primarily for use by the journal staff appear at the top of the list, above the most recent publications. This is because we did not think of storing them at our site until after the first issue was finished).

One solution to this problem, of course, might be to establish different communities for individual topics: a community for each issue, perhaps, plus an additional one for journal meta-documents and documentation. The main problem with this is that there is currently no way of associating different communities with each other in Zenodo. That is to say, this approach would allow the grouping of articles in individual issues, but not associate different issues with the journal as a whole. It is also strongly discouraged by Zenodo itself (Paolo Manghi, personal communication). OpenAIRE Connect, which will allow for collections of objects to be treated as citable research objects in their own right may ultimately provide a solution to this problem.

\section{Wordpress site}

The second dissemination route is via a free Wordpress site hosted by Wordpress.com (Graduate Student Association [University of Lethbridge], 2017a) (see Figure 5).
Figure 4: The front page of the Meeting of the Minds Zenodo Community

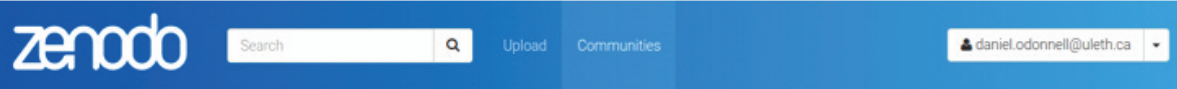

Meeting of the Minds Graduate Student Journal at the University of Lethbridge (ISSN: 2560-6670)
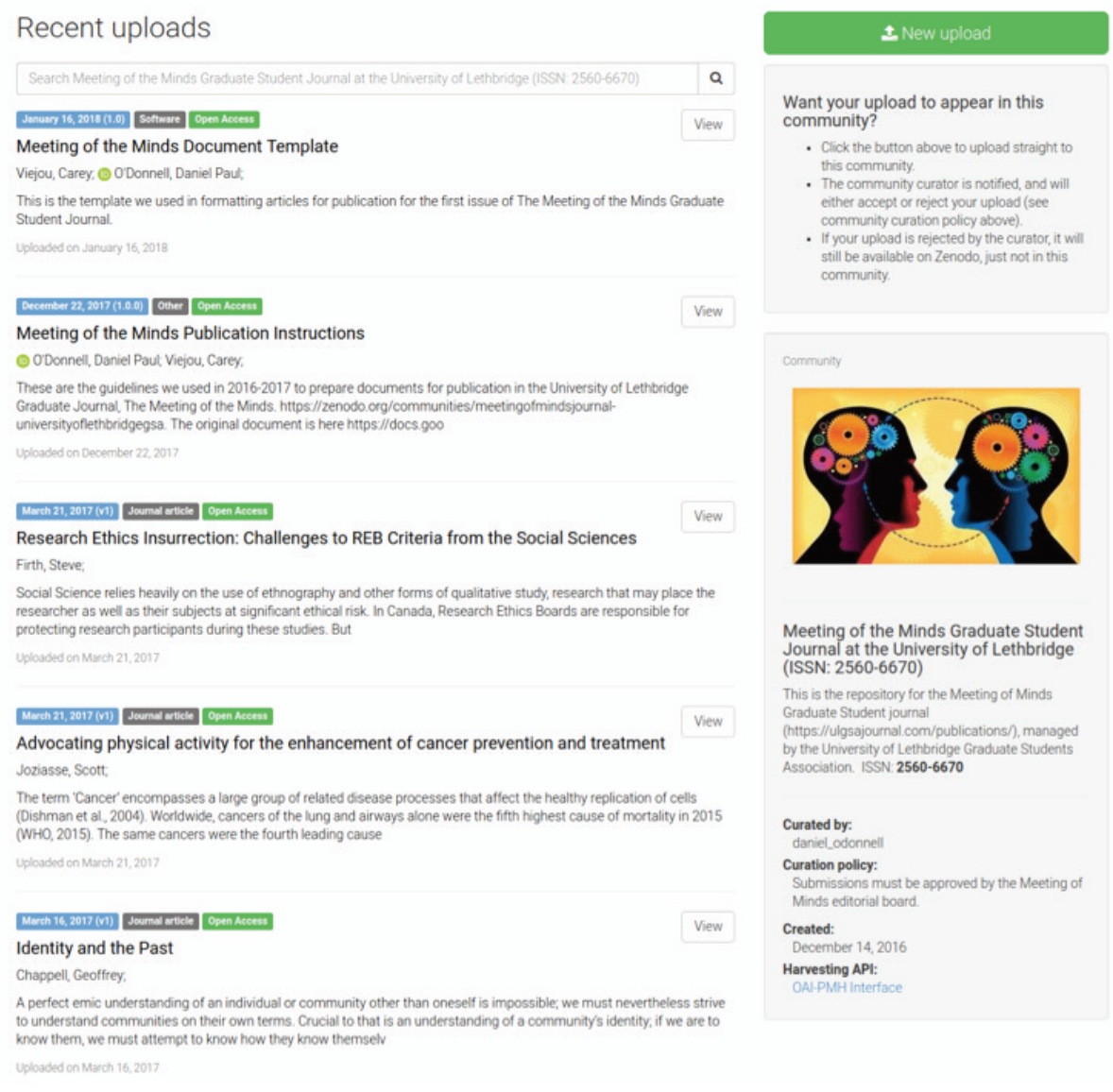

O’Donnell, Viejou, Chow, Dohms, Esau, Firth, Graham, McKinnon, Morrison, Parsons, Rieger, Spiric, Toth, Ueland. (2018). Zombie Journals: Designing a Technological Infrastructure for a Precarious Journal. Scholarly and Research Communication, 9(2): 0201296, 20 pp. 
Scholarly and Research

Communication

VOLUME 9 / ISSUE 2 / 2018
Figure 5: Branded Wordpress site

Meeting of the Minds Graduate Journal

A Publication of the University of Lethbridge's Graduate student Association

Home About $\sim$ Publications FAQ Contact

\section{Call for Submissions}

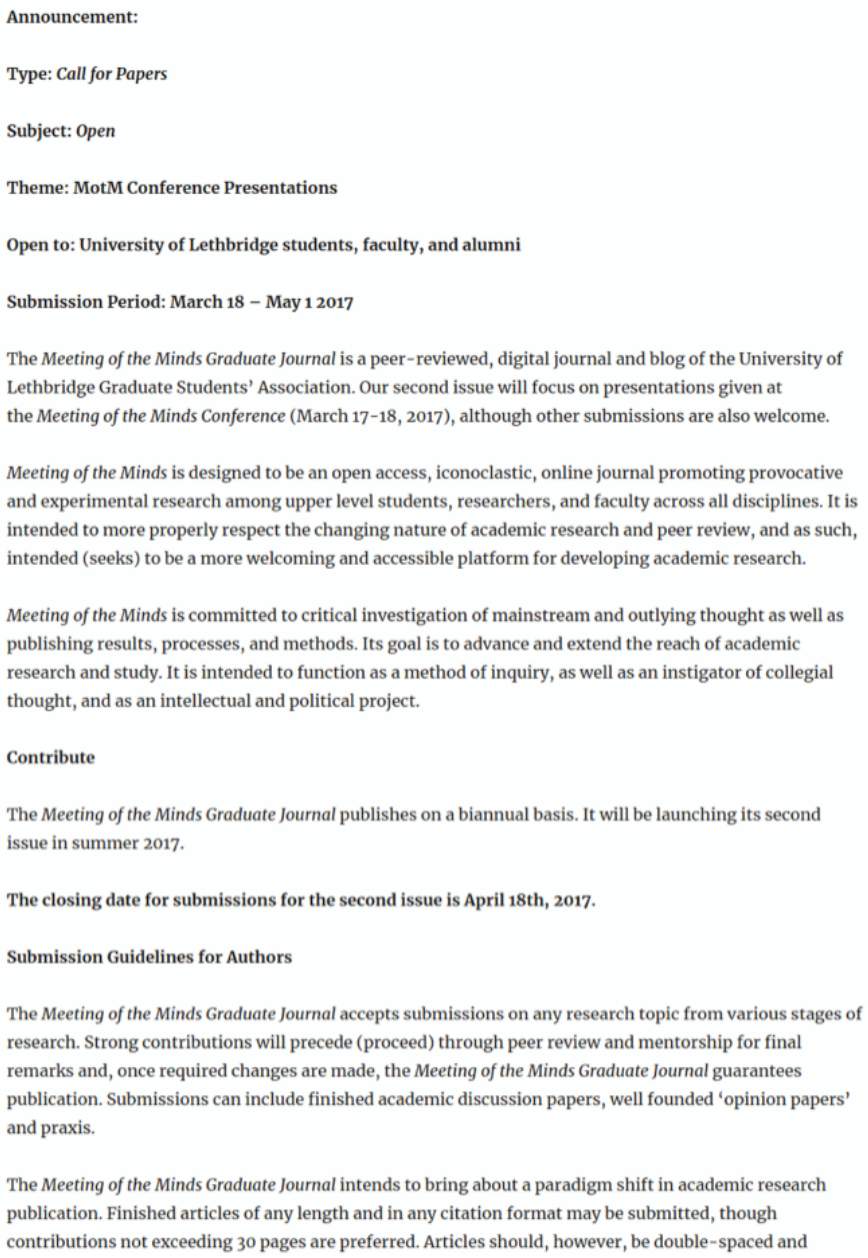

The Meeting of the Minds Graduate Journal accepts submissions on any research topic from various stages of research. Strong contributions will precede (proceed) through peer review and mentorship for final remarks and, once required changes are made, the Meeting of the Minds Graduate Journal guarantees publication. Submissions can include finished academic discussion papers, well founded 'opinion papers' and praxis.

The Meeting of the Minds Graduate Journal intends to bring about a paradigm shift in academic research publication. Finished articles of any length and in any citation format may be submitted, though contributions not exceeding 30 pages are preferred. Articles should, however, be double-spaced and

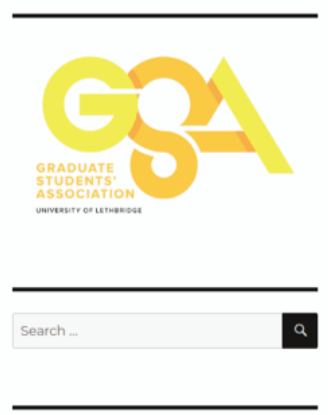

THE ELEVATOR

Meeting of the Minds is an biannual openjournal sponsored by the University of serves as a scholarly forum for graduate students at the University of Lethbridge to share and critically discuss their research. ISSN 2560-6670

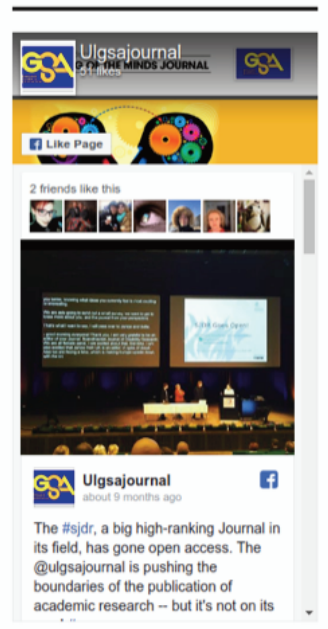
Lethbridge's Graduate Student Association.

This is a more specifically branded site for the journal, established by the editorial board early on out of a concern that the Zenodo site might not emphasize the connection to the $\mathrm{U}$ of $\mathrm{L}$ sufficiently. The site has its own branded URL, https://ulgsajournal.com/, which the GSA has agreed to support for the long term. The use of Wordpress with its ability to provide structure to posts also allows the grouping of articles by issues. On the other hand, for all the reasons discussed above (see "Blog/content management systems"), this site is both less permanent and more difficult to maintain than the "publish and forget" Zenodo site. As indicated in Figure 1, Zenodo acts as the document server that serves out the PDFs provided at the Wordpress site, further emphasizing the secondary nature of this collection.

\section{Conclusion}

The Meeting of the Minds journal is an experiment in student-led scholarly

O’Donnell, Viejou, Chow, Dohms, Esau, Firth, Graham, McKinnon, Morrison, Parsons, Rieger, Spirić, Toth, Ueland. (2018). Zombie Journals: Designing a Technological Infrastructure for a Precarious Journal. Scholarly and Research Communication, 9(2): 0201296, 20 pp. 
communication. Similar to many student journals, the set up and development focused on providing student editors and authors with a professional development experience that mirrors, as closely as possible, the demands of the discipline(s) they are apprenticing in.

At the same time, however, the students and faculty advisors responsible for setting up the journal felt an ethical duty to plan for the potential failure of the enterprise. As a primarily masters-level graduate school, the $\mathrm{U}$ of $\mathrm{L}$ does not have the opportunities for continuity found in programs with a stronger $\mathrm{PhD}$ and postdoctoral focus. At the $\mathrm{U}$ of L, most students have completed their programs and moved on to other activities (and, if they have stayed in academia, usually to other institutions) in 18 to 24 months. This means that we had to plan for both a very high turnover in participation and the possibility that future generations of students would not share our enthusiasm for the publication. At the same time, it necessitated finding a way to ensure the long-term preservation and discoverability of the articles that were published without having to rely on the willingness of these future generations to preserve our work.

After investigating and rejecting a number of common solutions for the publication of scholar-led journals, the editorial team developed a method of publication that satisfied all the design requirements, based entirely on off-the-shelf software and tools. The production method uses common word processors; freely available and strongly supported international tools are used to ensure the continuing discoverability of the published articles; and articles are published and archiveed using free services including Zenodo (the EU supported Open Science platform) and Wordpress.com, a commercial site that offers free hosting, albeit without guaranteed archiving. With the exception of the Wordpress site, the terms of use for which allow the host to terminate the site without notice, all of these tools are guaranteed to be stable and (relatively) permanent. Zenodo (n.d.) guarantees preservation for "the lifetime of the repository," which it defines as "currently the lifetime of the host laboratory CERN, which currently has an experimental programme defined for the next 20 years at least." The result is a true "publish and forget" system that guarantees the continued survival and discoverability of the Meeting of the Minds journal without relying on future generations' willingness to continue to support the work.

\section{Contributors}

The corresponding author is dpod. Authorship is alphabetical after the drafting author and principal technical lead. Author contributions, described using the CASRAI CRedIT typology (Consortia Advancing Standards in Research Administration Information, 2018), are as follows:

Conceptualization: pe, dpod, cv

Methodology: pe, dpod, cv

Software: dpod, cv

Investigation: sc, kd, pe, sf, rg, jm, rp, cr, vs, et, cv, ku, dpod

Resources: ku,dpod

Writing - Original Draft Preparation: dpod

Writing - Review \& Editing: dpod,kd,pe,rg,sf
VOLUME 9 / ISSUE 2 / 2018 


\section{Scholarly and Research}

Communication

VOLUME 9 / ISSUE $2 / 2018$
Supervision: rg,dpod

Project Administration: rg,dpod

Funding Acquisition: ku,dpod

\section{Notes}

1. In arguing that OJS was not viable for our journal, we are in no way implying that it is not an excellent system or very commonly an appropriate system to use for scholar-led and/or amateur journals. What we are arguing is that in our particular case, with high turnover, little (and often no) money, and no guarantee as to local expertise or support, the system was too specialized and required too much future maintenance to be viable (for a similar observation from a different perspective, see Maxwell, 2007, 2009).

2. We say "almost" because in the current model, communities are not, formally speaking, citable scholarly objects similar to the documents they contain (e.g., they are not assigned DOIs and have minimal metadata). OpenAIRE Connect is a new project that among other things proposes treating collections as scholarly objects in their own right (Koulocheri, 2017; Natalia Menola and Paolo Manghi, personal communication).

3. Objects can be accessed directly (i.e., without this landing page) using the URL found under the "Name" field (bottom left).

\section{References}

Automattic Inc. (2014, September 19). Terms of service. URL: https://en.wordpress.com/tos/ [November 6, 2017].

Bankier, Jean-Gabriel, \& Perciali, Irene. (2008). The Institutional repository rediscovered: What can a university do for open access publishing? Serials Review, 34(1), 21-26. URL: https://doi.org /10.1080/00987913.2008.10765147 [April 9, 2018].

Consortia Advancing Standards in Research Administration Information. (2018, January 29). “CRediT." CASRAI. URL: http://ref.casrai.org/CRediT [April 9, 2018].

Cowan, Sandra A. (2013). Open Access journal incubator at University of Lethbridge library. In Allison P. Brown (Ed.), Library publishing toolkit (pp. 179-186). Geneseo, NY: IDS Project Press. URL: https://www.uleth.ca/dspace/bitstream/handle/10133/4595/Cowan\%202013\%20open \%2olibpubtool.pdf? sequence $=1$ [April 9, 2018].

Esau, Paul, Viejou, Carey, Chow, Sylvia, Dohms, Kimberly, Firth, Steven, McKinnon, Jarret, Morrison, Dorthea, Parsons, Reed, Rieger, Courtney, Spiric, Vanja, Toth, Elaine, Ueland, Kayla, \& O’Donnell, Daniel Paul. (2018, March 27). "Let's start a journal!" The multidisciplinary graduate student journal as educational opportunity. University of Lethbridge. URL: https://doi.org/10.5281 /zenodo.1208710 [April 9, 2018].

Eve, Martin Paul. (2012, July 10). Starting an open access journal: A step-by-step guide part 1. URL: https://www.martineve.com/2012/07/10/starting-an-open-access-journal-a-step-by-step-guide -part-1/ [November 1, 2017].

Fortney, Katie, \& Gonder, Justin. (2015, December 1). A social networking site is not an open access repository. URL: http://osc.universityofcalifornia.edu/2015/12/a-social-networking-site-is-not -an-open-access-repository/ [March 27, 2018].

Ghosh, Abhishek. (2014). WordPress as academic journal: Needed plugins. The Customize Windows. URL: https://thecustomizewindows.com/2014/o6/wordpress-academic-journal-needed-plugins/ [April 9, 2018].

O’Donnell, Viejou, Chow, Dohms, Esau, Firth, Graham, McKinnon, Morrison, Parsons, Rieger, Spirić, Toth, Ueland. (2018). Zombie Journals: Designing a Technological Infrastructure for a Precarious Journal. Scholarly and Research Communication, 9(2): 0201296, 20 pp. 
Government of Alberta. (2007, December 31). Comprehensive Academic and Research Institutions. Alberta Innovation and Advanced Education. URL: http://advancededucation.alberta.ca/postsecondary/institutions/public/types/cari/. [April 9, 2018].

Graduate Student Association [University of Lethbridge]. (2017a). Meeting of the Minds Graduate Journal. URL: https://ulgsajournal.com/ [December 13, 2017].

Graduate Student Association [University of Lethbridge]. (2017b). Meeting of the Minds Graduate Student Journal at the University of Lethbridge. URL: https://zenodo.org/communities $/$ meetingofmindsjournal-universityoflethbridgegsa/?page $=1 \&$ size $=20$ [December 13, 2017].

Hendricks, Ginny (n.d.). Fees - Crossref. URL: https://www.crossref.org/fees/ [January 15, 2018].

Koulocheri, Eleni. (2017, January 25). About OpenAIRE-Connect. URL: https://www.openaire.eu /connect [January 16, 2018].

Leubsdorf, Carl, Jr. (2011). Annotum: An open-source authoring and publishing platform based on WordPress. Bethesda, MD: National Center for Biotechnology Information. URL: https://www .ncbi.nlm.nih.gov/books/NBK63828/ [April 9, 2018].

Library and Archives Canada. (2017, December 12). ISSN Canada. URL: http://www.bac-lac.gc.ca /eng/services/issn-canada/Pages/issn-canada.aspx (Original work published April 29, 2013) [January 15, 2018].

Maxwell, John W. (2007). Extending OJS into small magazines: The OMMM Project. First Monday, 12(10). URL: http://journals.uic.edu/ojs/index.php/fm/article/view/1962/1839 [April 9, 2018].

Maxwell, John W. (2009). The OMMM project: Toward a collaborative editorial workflow. Scholarly and Research Communication, 1(1). URL: http://src-online.ca/index.php/src/article/view/5/27 [April 9, 2018].

O’Donnell, Daniel Paul, Hobma, Heather, Cowan, Sandra A., Ayers, Gillian, Bay, Jessica, Swanepoel, Marinus, Merkley, Wendy, Devine, Kelaine, Dering, Emma, \& Genee, Inge. (2015). Aligning open access publication with research and teaching missions of the public university: The case of the Lethbridge Journal Incubator (if "if"s and "and"s were pots and pans). The Journal of Electronic Publishing: JEP, 18(3). URL: http://quod.lib.umich.edu/cgi/t/text/idx/j/jep/3336451.0018.309/_ aligning-open-access-publication-with-research-and-teaching?rgn=main;view=fulltext [April 9, 2018].

O’Donnell, Daniel Paul, \& Viejou, Carey. (2017). Meeting of the Minds: Publication instructions (Version 2016-2017). Lethbridge, AB: University of Lethbridge. https://doi.org/10.5281 /zenodo.1127750 [April 9, 2018].

Open Library of Humanities. (n.d.). Home. URL: https://www.openlibhums.org/ [November 6, 2017]. sbstarr, Laxmin, \& PixelatorOfTime. (2015, July 27). Scientific Journal in WordPress $\bullet r /$ Wordpress.

URL: https://www.reddit.com/r/Wordpress/comments/3es8k1/scientific_journal_in_wordpress/ [November 6, 2017].

Scholastica. (n.d.). Efficient. Affordable. Liberating. Welcome to your modern academic journal management system. URL: https://scholasticahq.com/ [November 6, 2017].

Solvitor LLC. (n.d.). Annotum final release. URL: https://annotum.org/site\%2oupdates/2016/11/22 /annotum-final-release.html [November 6, 2017].

Suber, Peter. (2013, December 16). Open access overview (definition, introduction). URL: http://legacy .earlham.edu/ peters/fos/overview.htm [July 8, 2014].

Swanepoel, Marinus, Kehoe, Inba, Hohner, Michael, Shepstone, Carol, Vanderjagt, Leah, Wakaruk, Amanda, Waller, Andrew, \& Winter, Christina. (2015). Developing a community of practice: Report on a survey to determine the scholarly communication landscape in western Canada. URL: https://www.uleth.ca/dspace/handle/10133/4784 [April 9, 2018].

O’Donnell, Viejou, Chow, Dohms, Esau, Firth, Graham, McKinnon, Morrison, Parsons, Rieger, Spiric, Toth, Ueland. (2018). Zombie Journals: Designing a Technological Infrastructure for a Precarious Journal. Scholarly and Research Communication, 9(2): 0201296, 20 pp.
Scholarly and Research Communication

VOLUME 9 / ISSUE 2 / 2018 
Scholarly and Research

\section{Communication}

VOLUME 9 / ISSUE 2 / 2018
University of Lethbridge. (2017). Fall 2017. URL: http://www.uleth.ca/analysis/content/view-facts [December 13, 2017]

Viejou, Carey, \& O'Donnell, Daniel Paul. (2018). Meeting of the minds: Document template. URL: https://doi.org/10.5281/zenodo.1149678 [April 9, 2018].

Zenodo. (n.d.). About Zenodo. URL: http://about.zenodo.org/ [January 16, 2018]. 